\title{
Suppression of Relative Intensity Noise in Fiber-Optic Raman Polarizers
}

\author{
Victor V. Kozlov and Stefan Wabnitz, Member, IEEE
}

\begin{abstract}
We propose a method for the suppression of relative intensity noise (RIN) at the output of a Raman polarizer, a special type of Raman amplifier that transforms an input weak beam of unpolarized light into an highly polarized amplified beam towards its output. We show that the RIN which is induced by polarization-dependent gain is suppressed whenever the Raman polarizer works in the depleted pump regime.
\end{abstract}

Index Terms-Optical fibers, polarization, Raman scattering.

$\mathbf{T}$ YPICAL fiber optic Raman amplifiers operate in a regime such that polarization-dependent gain (PDG) is minimal, leading to approximately the same value of gain independently of the state of polarization (SOP) of the input signal beam. For many applications the insensitivity of Raman gain to the input signal SOP is a key advantage. The SOP of the signal beam at the output of these Raman amplifiers mostly depends on the input signal SOP only. Therefore whenever the signal beam is initially unpolarized, the output signal beam will also be unpolarized.

On the other hand, several optical devices (such as parametric amplifiers) would require highly polarized light emerging from a Raman amplifier. Recently proposed in [1], the so-called Raman polarizers combine Raman amplification with the simultaneous repolarization of light. Namely, the outcoming signal beam is highly polarized, even in cases when the Raman polarizer is fed by unpolarized light. With respect to the simple alternative provided by a typical Raman amplifier followed by a passive polarizer, the Raman polarizer offers the possibility of all-optical control of the SOP of the output signal beam by means of varying the SOP of the pump beam. Raman polarizers require for their operation a highly selective PDG, so that a particular signal SOP is maximally amplified, whereas its orthogonal polarization component experiences virtually no amplification. This property makes a Raman polarizer analogous to a conventional passive polarizer. In particular, the output of a Raman polarizer suffers from strong RIN when fed by unpolarized light. In this Letter we demonstrate that

Manuscript received January 24, 2011; revised April 02, 2011; accepted May 14, 2011. Date of publication May 19, 2011; date of current version July 15 , 2011. This work was carried out in the framework of the "Scientific Research Project of Relevant National Interest" (PRIN 2008) titled "Nonlinear cross-polarization interactions in photonic devices and systems" (POLARIZON).

V. V. Kozlov is with the Department of Information Engineering, University of Brescia, Brescia 25123, Italy, and also with the Department of Physics, Saint Petersburg State University, Petrodvoretz, Saint Petersburg, 198504, Russia (e-mail: victor.kozlov@email.com).

S. Wabnitz is with the Department of Information Engineering, University of Brescia, Brescia 25123, Italy (e-mail: stefano.wabnitz@ing.unibs.it).

Color versions of one or more of the figures in this letter are available online at http://ieeexplore.ieee.org.

Digital Object Identifier 10.1109/LPT.2011.2157119 operating a Raman polarizer in the depleted regime may result in efficient suppression of the output RIN. Note that this type of RIN is induced by the PDG, and it should not be confused with the RIN that is transferred to the signal from the fluctuating pump beam, [2], [3].

From the practical point of view, fiber optic Raman polarizers differ from typical Raman amplifiers in that a peculiar type of gain fiber should be selected. Namely, a Raman polarizer requires a low polarization mode dispersion (PMD) fiber, i.e., with PMD coefficients lower than $0.02 \mathrm{ps} / \sqrt{\mathrm{km}}$, see [1], [4], [5]. The lower the PMD coefficient, the greater the PDG, the better the performance of the Raman polarizer, the worse the RIN induced over the signal. For practically achievable values of the PMD coefficient, Raman polarizers require pump power levels of the order of $5 \mathrm{~W}$. Given the high gain of a Raman polarizer (which is ideally twice the gain of a typical Raman amplifier using relatively high PMD fibers) at the quoted high pump power levels, the associated length of a standard telecommunication gain fiber remains below $2 \mathrm{~km}$. Note that the pump power requirements are eased when using gain fibers with yet lower values of the PMD coefficient. Indeed experiments in [6] were performed with a PMD coefficient as low as $0.0037 \mathrm{ps} / \sqrt{\mathrm{km}}$ and pump power levels as low as $2 \mathrm{~W}$.

Raman polarizers can operate in the copropagating pump regime as well as (see [5]) in the counter-propagating pump configuration. Although computer-friendly theories of the $\chi^{(3)}$-interaction in telecommunication (i.e., randomly birefringent) fibers for both geometries were recently developed in [4], [5], here we shall limit ourselves to the copropagating case. Detailed derivations can be found in [5]: here we only provide the final equation for the evolution of the Stokes vector $S^{(s)}=\left(S_{1}^{(s)}, S_{2}^{(s)}, S_{3}^{(s)}\right)$ of the signal beam:

$$
\begin{aligned}
\left(\partial_{z}+\right. & \left.\beta^{\prime}\left(\omega_{s}\right) \partial_{t}\right) S^{(s)} \\
= & -\alpha_{s} S^{(s)}+\gamma\left(\omega_{s}\right) \\
& \times\left(S^{(s)} \times \boldsymbol{J}_{S}^{(s)}(z) S^{(s)}+S^{(s)} \times \boldsymbol{J}_{X}(z) S^{(p)}\right) \\
& +\epsilon_{s} g_{0}\left(S_{0}^{(p)} J_{R 0} S^{(s)}+S_{0}^{(s)} \boldsymbol{J}_{R}(z) S^{(p)}\right),
\end{aligned}
$$

where $S_{0}^{(p, s)}$ is the pump/signal power. The Stokes vector components are written in terms of the two polarization components $V_{s 1}$ and $V_{s 2}$ of the slowly varying signal field in the appropriate reference frame, as $S_{1}^{s}=V_{s 1} V_{s 2}^{*}+V_{s 1}^{*} V_{s 2}, S_{2}^{s}=$ $i\left(V_{s 1}^{*} V_{s 2}-V_{s 1} V_{s 2}^{*}\right), S_{3}^{s}=\left|V_{s 1}\right|^{2}-\left|V_{s 2}\right|^{2}$. Similar equations and definitions (with labels $p$ and $s$ interchanged) hold for the pump beam. $\gamma\left(\omega_{s}\right)$ is the Kerr coefficient of the fiber at frequency $\omega_{s}$ of the signal beam; $g_{0}$ is the Raman gain coefficient; $\beta^{\prime}\left(\omega_{s}\right)$ is the inverse group velocity of the signal beam; $\alpha_{s}$ is 
the fiber attenuation coefficient; $\epsilon_{s}=1 ; \epsilon_{p}=-\omega_{p} / \omega_{s}$. Details for the computation of the diagonal self- and cross-polarization modulation matrices $\boldsymbol{J}_{S}^{(s)}(z)$ and $\boldsymbol{J}_{X}(z)$, diagonal Raman matrix $\boldsymbol{J}_{R}(z)$, and coefficient $J_{R 0}$ can be found in [4], [5].

We self-consistently numerically solved the equations for the pump and signal Stokes vectors with the following parameters of the telecommunication fiber: the beat length $L_{B}=100 \mathrm{~m}$, the PMD coefficient $D_{p}=0.0073 \mathrm{ps} / \sqrt{\mathrm{km}}$, the attenuation coefficient $\alpha_{s}=\alpha_{p}=0.2 \mathrm{~dB} / \mathrm{km}$, the Raman gain coefficient $g_{0}=0.6(\mathrm{~W} \cdot \mathrm{km})^{-1}$, the fiber length $1.5 \mathrm{~km}$. Also $\lambda_{s}=2 \pi c / \omega_{s}=1.55 \mu \mathrm{m}$ and $\lambda_{p}=2 \pi c / \omega_{p}=1.45 \mu \mathrm{m}$, with $c$ as phase speed of light in vacuum. In all simulations, the input power of the CW pump beam $S_{0}^{(p)}(z=0)=8 \mathrm{~W}$, its SOP was fixed at $(0,1,0)$. The input $\mathrm{CW}$ signal power $S_{0}^{(s)}(z=0, t)$ was varied between $0.05 \mathrm{~mW}$ and $10 \mathrm{~mW}$. Let us first consider input signals with a time-oscillating SOP as defined by the relations

$$
\begin{aligned}
& S_{1}^{(s)}(z=0, t)=S_{0}^{(s)}(0, t) \sin \left[\alpha_{1}(t)\right] \cos \left[\alpha_{2}(t)\right], \\
& S_{2}^{(s)}(z=0, t)=S_{0}^{(s)}(0, t) \sin \left[\alpha_{1}(t)\right] \sin \left[\alpha_{2}(t)\right], \\
& S_{2}^{(s)}(z=0, t)=S_{0}^{(s)}(0, t) \cos \left[\alpha_{1}(t)\right] .
\end{aligned}
$$

Here $\alpha_{1}(t)=0.0506 \omega_{m} t, \alpha_{2}=0.0706 \omega_{m} t$, where $\omega_{m}=$ $c / L_{N L}, L_{N L}=\left[\gamma\left(\omega_{s}\right) S_{0}^{(p)}\right]^{-1}=125 \mathrm{~m}$. Note that the specific choice of the modulation frequency $\omega_{m}$ has no influence on the performance of the Raman polarizer, because its response is instantaneous. For a sufficiently long observation time $T$, such that $\omega_{m} T \gg 1$, the time variations imposed by (2)-(4) mimics the behavior of unpolarized light.

The first example in Fig. 1(a) and (b) shows how the Raman polarizer repolarizes light. Input temporal variations of the polarization of the signal beam disappear at the device output, where the signal SOP is strictly aligned along the $(0,1,0)$ direction. As expected, the output signal SOP coincides with the SOP of the pump beam, see [4].

From the other hand, input signal polarization fluctuations coupled with PDG lead to RIN at the signal output. In fact the output power of the signal beam shown in Fig. 2(c) exhibits strong PDG-induced temporal variations. Such output intensity temporal variations also appear with an input power-stabilized $\mathrm{CW}$ signal beam. As it can be seen, the resulting RIN is well pronounced at low input signal powers, while it is heavily suppressed as the signal intensity grows larger. For the current parameters, the weakest input power in Fig. 1(c) corresponds to the nearly undepleted regime, while the strongest input power corresponds to the well depleted regime. This is the main message of this Letter, namely that the effect of pump depletion effectively suppresses the output RIN caused by PDG.

In order to quantify the effect of RIN, we computed two quantities of interest, both of which are shown in Fig. 2(a) and (b). The first quantity is the output signal mean power $\left\langle S_{0}^{(s)}(L, t)\right\rangle_{T}$, which is the signal power averaged over a relatively long time interval $T$ (e.g., $\omega_{m} T=10^{4}$ ). Time $T$ is such that when averaged over this interval the input beam behaves as unpolarized light (i.e., its degree of polarization is vanishingly small). On this time scale the mean output power is steady in time: its value is shown in Fig. 2(a) as a function of the $\mathrm{CW}$ input power.
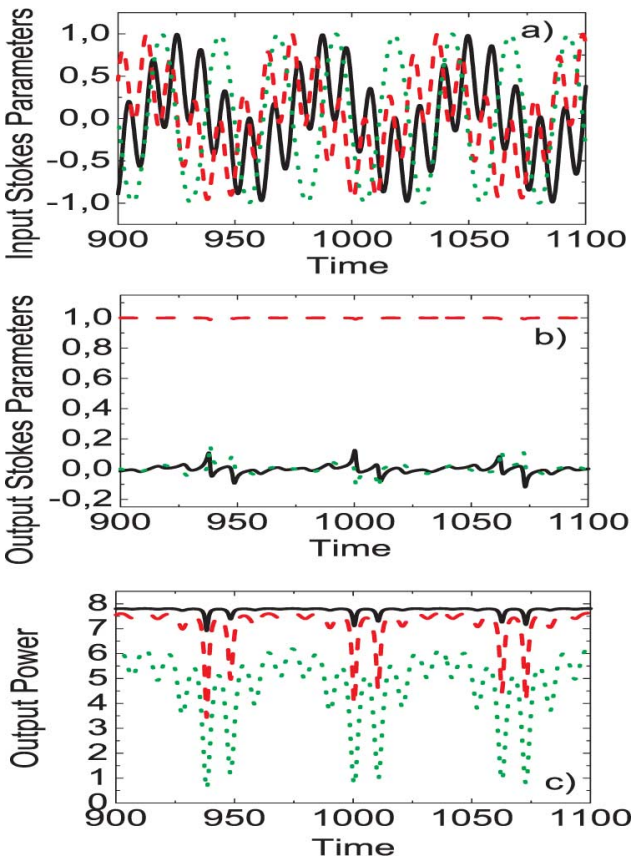

Fig. 1. Input and output characteristics of a Raman polarizer in the course of time. (a) Input: $S_{1}^{+}(z=0)$ (black solid line), $S_{2}^{+}(z=0)$ (red dashed line), $S_{3}^{+}(z=0)$ (green dotted line); (b) output: $S_{1}^{+}(z=L)$ (black solid line), $S_{2}^{+}(z=L)$ (red dashed line), $S_{3}^{+}(z=L)$ (green dotted line); (c) output: $S_{0}^{+}(L)$ (in watts) for $S_{0}^{+}(0)=10 \mathrm{~mW}$ (black solid line), $1 \mathrm{~mW}$ (red dashed line), $0.1 \mathrm{~mW}$ (green dotted line). For (a), (b) $S_{0}(z=0, t)=0.1 \mathrm{~mW}$.
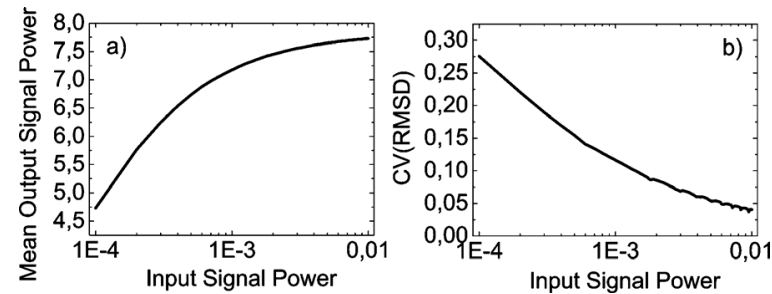

Fig. 2. (a) Mean power (in watts), and (b) the deviation from the mean characterized by the CV(RMSD), both quantities relating to the output signal beam. The input SOP varies with time as dictated by (2)-(4). The input signal power (in watts) is steady in time. The observation time $T=10^{4} w_{m}^{-1}$.

For the characterization of RIN we may introduce the root mean square deviation (RMSD) as

$$
\operatorname{RMSD}=\sqrt{\frac{1}{T} \int_{t_{0}}^{t_{0}+T} d t\left[S_{0}^{(s)}(L, t)-\left\langle S_{0}^{(s)}(L, t)\right\rangle_{T}\right]^{2}},
$$

and derive from it the coefficient of variation of the RMSD: namely, CV $(\mathrm{RMSD})=\mathrm{RMSD} /\left\langle S_{0}^{(s)}(L, t)\right\rangle_{T}$, which is a dimensionless quantity. Fig. 2(b) quantifies the RIN suppression effect with the increase of the signal power: the lower the CV (RMSD), the lesser the RIN.

Output RIN suppression in the depleted pump regime also takes place for input signal beams with time fluctuating (e.g., harmonically) power:

$$
S_{0}^{(s)}(0, t)=P\left[1+A_{m} \sin \left(0.2 \omega_{m} t\right)\right],
$$

where $A_{m}$ is the modulation amplitude. As before, fluctuations of the signal SOP are also imposed on the input signal beam. 


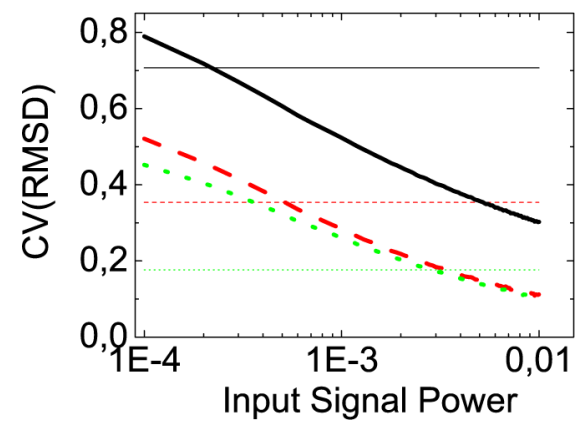

Fig. 3. CV (RMSD) versus input signal power (in watts) for three intensity modulation amplitudes of the input signal: $A_{m}=1$ (black solid line); 0.5 (red dashed line); 0.25 (green dotted line). Input—-thin lines; output—-thick lines.

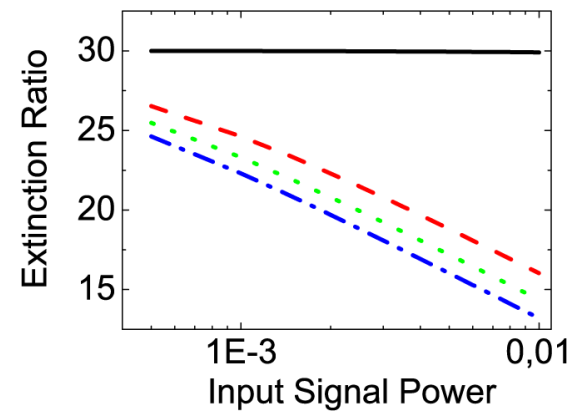

Fig. 4. Extinction ratio (in decibels) of the output signal as a function of input signal power (in watts) for four SOPs of the input signal beam: $(0,-1,0)$ (black solid line); $(0,0,1)$ (red dashed line); $(1 / 2)(1,1, \sqrt{2})$ (green dotted line); $(0$. $1,0)$ (blue dotted-dashed line). The extinction ratio of the input beam is $30 \mathrm{~dB}$ for all cases. Note that the blue dotted-dashed curve is obtained for the case of maximal Raman gain and therefore it provides the largest degradation of the extinction ratio.

Even in this relatively more complex case, the operation of the Raman polarizer in the depleted (or saturated gain) regime leads to significant RIN suppression at the device output, as demonstrated by Fig. 3.

So far we discussed the amplification of $\mathrm{CW}$ signals, for which the signal-to-noise ratio (SNR) is not an issue. With pulsed signals, as in the flow of bits in a telecommunication link, operation of the Raman polarizer in the depleted regime leads to a degradation of the SNR. For example, in Fig. 4 we show the degradation of the extinction ratio with the increase of the signal power for a sequence of square pulses.

For a more quantitative analysis of the operation of a Raman polarizer as a function of the input signal power, we considered its action on a pseudorandom bit sequence (PRBS). We fixed the SOP of each bit in the PRBS input sequence, and varied it from bit to bit as described by (2)-(4) (for this example, the input signal SOP is changed in a step-like mannner in time intervals exactly equal to the duration of one bit). We then computed the $Q$-factor and associated bit-error rate (BER) (using the Gaussian approximation) by means of a standard receiver implemented in the Virtual Photonics Transmission Maker soft-
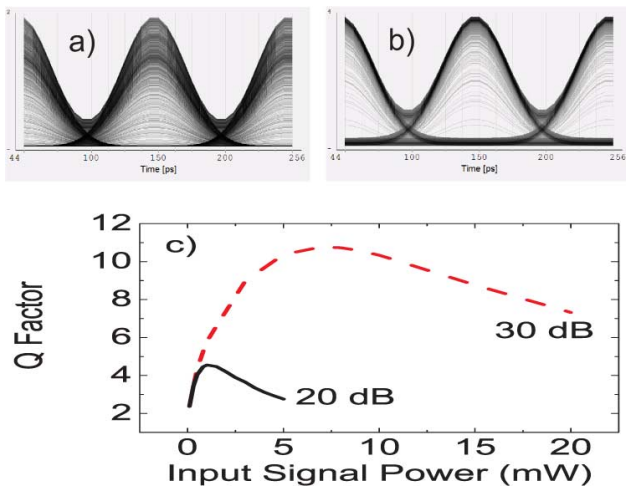

Fig. 5. Eye diagrams for two different input powers of the signal beam: (a) $0.1 \mathrm{~mW}$; (b) $7 \mathrm{~mW}$. (c) $Q$-factor of the output bit sequence as the function of input power for two values of the extinction ratio shown on the figure.

ware. For a $10 \mathrm{~Gb} / \mathrm{s}, 2^{12}$ PRBS composed of square-like return-to-zero pulses with 50 percent duty cycle, extinction ratios 20 and $30 \mathrm{~dB}$, and different power levels, the $Q$-factor exhibits a characteristic hump structure as a function of the input signal power, see Fig. 5. The optimal $Q$ value results from a trade-off between the desirable equalization of the power levels of the ones, and the simultaneous unwanted degradation of zeroes, as the input power grows larger. Note that for the case of a $30 \mathrm{~dB}$ extinction ratio, the BER in the undepleted regime (below $0.1 \mathrm{~mW}$ ) is above 0.004 , while in the depleted regime for the optimal power level of $7 \mathrm{~mW}$ it drops down to $10^{-27}$. The significant degradation of the extinction ratio of the pulse sequence emerging from a Raman polarizer operating in the depleted regime shows that Raman polarizer can be used in a transmission line only once, for instance near its end.

In conclusion, we proposed a method for the suppression of the signal RIN at the output of a Raman polarizer which is induced by its PDG. The method works equally well for both CW and for pulsed signals.

\section{REFERENCES}

[1] M. Martinelli, M. Cirigliano, M. Ferrario, L. Marazzi, and P. Martelli, "Evidence of Raman-induced polarization pulling," Opt. Express, vol. 17, pp. 947-955, 2009.

[2] C. R. S. Fludger, V. Handerek, and R. J. Mears, "Pump to signal RIN transfer in Raman fiber amplifiers," J. Lightw. Technol., vol. 19, no. 8, pp. 1140-1148, Aug. 2001.

[3] B. Bristiel, S. Jiang, P. Gallion, and E. Pincemin, "New model of noise figure and RIN transfer in fiber Raman amplifiers," IEEE Photon. Technol. Lett., vol. 18, no. 8, pp. 980-982, Apr. 15, 2006.

[4] V. V. Kozlov, J. Nun̄o, J. D. Ania-Castañón, and S. Wabnitz, "Theory of fiber optic Raman polarizers," Op. Lett., vol. 35, pp. 3970-3972, 2010.

[5] V. V. Kozlov, J. Nun̄o, J. D. Ania-Castañón, and S. Wabnitz, "Theoretical study of fiber-based Raman polarizers with counterpropagating beams," J. Lightw. Technol., vol. 29, no. 3, pp. 341-347, Feb. 1, 2011.

[6] M. Ferrario, V. Gilardone, P. Martelli, L. Marazzi, and M. Martinelli, "Effective all-optical polarization control induced by Raman nonlinear amplification," in Proc. ECOC 2010, Torino, Italy, Sep. 2010, pp. 19-23, Paper 19. 